\title{
Erratum: Energy flow in quantum critical systems far from equilibrium
}

M. J. Bhaseen, Benjamin Doyon, Andrew Lucas and Koenraad Schalm

Nature Physics 11, 509-514 (2015); published online 4 May 2015; corrected after print 3 September 2015.

In the print and PDF versions of this Article originally published the digital object identifier contained a typographical error and should have read $10.1038 /$ nphys 3320 . This error has now been corrected in the online versions. 